\title{
Multimode interaction in axially excited cylindrical shells
}

\author{
F. M. A. Silva ${ }^{2}$, L. Rodrigues ${ }^{1}$, P. B. Gonçalves ${ }^{1}$, and Z. J. G. N Del Prado ${ }^{2}$ \\ ${ }^{1}$ Department of Civil Engineering, Pontifical Catholic University of Rio de Janeiro, Brazil \\ ${ }^{2}$ School of Civil Engineering, Federal University of Goiás, Brazil
}

\begin{abstract}
Cylindrical shells exhibit a dense frequency spectrum, especially near the lowest frequency range. In addition, due to the circumferential symmetry, frequencies occur in pairs. So, in the vicinity of the lowest natural frequencies, several equal or nearly equal frequencies may occur, leading to a complex dynamic behavior. So, the aim of the present work is to investigate the dynamic behavior and stability of cylindrical shells under axial forcing with multiple equal or nearly equal natural frequencies. The shell is modelled using the Donnell nonlinear shallow shell theory and the discretized equations of motion are obtained by applying the Galerkin method. For this, a modal solution that takes into account the modal interaction among the relevant modes and the influence of their companion modes (modes with rotational symmetry), which satisfies the boundary and continuity conditions of the shell, is derived. Special attention is given to the 1:1:1:1 internal resonance (four interacting modes). Solving numerically the governing equations of motion and using several tools of nonlinear dynamics, a detailed parametric analysis is conducted to clarify the influence of the internal resonances on the bifurcations, stability boundaries, nonlinear vibration modes and basins of attraction of the structure.
\end{abstract}

\section{Introduction}

Cylindrical shells exhibit a dense frequency spectrum, especially near the lowest frequency range. In addition, due to the circumferential symmetry, frequencies occur in pairs. So, in the vicinity of the lowest natural frequencies, several equal or nearly equal frequencies may occur. This closeness is even greater when one considers a static compressive loading. This may lead to a strong nonlinear interaction among these modes under dynamic conditions, influencing thereby the response and safety of the shell. Although there are many papers on modal coupling and interaction in the presence of static loads, little is known on their influence on the dynamic stability of thin-walled cylindrical shells. So, when considering the effect of nonlinearity, there is the possibility of interaction between the different non-linear vibration modes, which can cause significant changes in the stability boundaries and bifurcation diagrams [1, 2]. However, no consistent modal solution taking into account the simultaneous effect of modal coupling plus modal interaction is found in literature [3, 4].

So the aim of the present work is (a) to deduce a consistent modal solution for a shell where two modes exhibits equal natural frequencies, retaining all modes that appear due to the nonlinear modal coupling and modal interaction, (b) obtain from this general expression a precise low order model for large amplitude vibrations, and (c) use this modal solution to study the nonlinear oscillations and instabilities of these cylindrical shells under axial loads. This modal solution is obtained by applying perturbation techniques, as suggested previously by the authors in $[5,6]$.

\section{Problem formulation}

\subsection{Shell equations}

Consider a perfect thin-walled circular cylindrical shell of radius $R$, length $L$, and thickness $h$. The shell is assumed to be made of an elastic, homogeneous, and isotropic material with Young's modulus $E$, Poisson ratio $v$ and density $\rho$. The axial, circumferential and lateral coordinates are denoted by, respectively, $x, \theta$ and $z$, and the corresponding displacements on the shell surface are in turn denoted by $u, v$, and $w$, as shown in figure 1 .

The nonlinear equations of motion based on the Von Karmán-Donnell shallow shell theory, in terms of a stress function $F$ and the transversal displacement $w$ are given by:

$$
\begin{aligned}
& \rho h \ddot{w}+\beta_{1} \rho h \omega_{0} \dot{w}+\beta_{2} D \nabla^{4} \dot{w}+D \nabla^{4} w= \\
& \frac{\left(F_{, \theta \theta}-P(t)\right)}{R^{2}} w_{, x x}-2 \frac{F_{, x \theta}}{R} \frac{w_{, x \theta}}{R}+F_{, x x}\left(\frac{w_{, \theta \theta}}{R^{2}}+\frac{1}{R}\right)
\end{aligned}
$$


$\frac{1}{E h} \nabla^{4} F=-\frac{1}{R} w_{, x x}-w_{, x x} \frac{w_{, \theta \theta}}{R^{2}}+\left(\frac{w_{, x \theta}}{R}\right)^{2}$

where $\beta_{1}$ and $\beta_{2}$ are, respectively, the linear viscous damping and the viscoelastic material damping coefficients, $D=E h^{3} / 12\left(1-v^{2}\right)$ is the flexural stiffness of the shell, $\omega_{0}$ is the lowest vibration frequency, $P(t)$ is the axial load and $\nabla^{4}$ is the bi-harmonic operator defined as $\nabla^{4}=\left[\partial^{2} / \partial x^{2}+\partial^{2} / R^{2} \partial \theta^{2}\right]^{2}$.

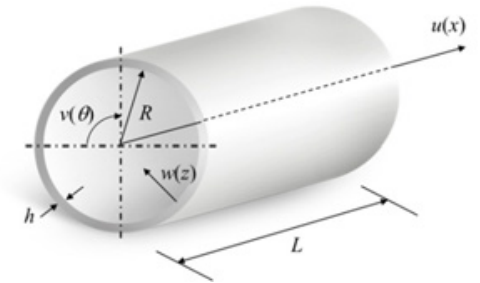

Fig. 1. Geometry and displacement field of the cylindrical shell.

The shell is subjected to a harmonic axial load, uniformly applied along the edges, of the form:

$P=P_{0}+P_{1} \cos \left(\omega_{f} t\right)$

For a simply-supported shell, the following boundary conditions must be satisfied in the transversal direction:

$w(0, \theta)=w(L, \theta)=0$ and $M_{x}(0, \theta)=M_{x}(L, \theta)=0$

In the foregoing, the following non-dimensional parameters have been introduced:

$W=\frac{w}{h} ; \varepsilon=\frac{x}{L} ; \tau=\omega_{0} t ; \Omega_{f}=\frac{\omega_{f}}{\omega_{0}}$

$\Gamma_{0}=\frac{P_{0}}{P_{\mathrm{CR}}} ; \Gamma_{1}=\frac{P_{1}}{P_{\mathrm{CR}}}$

Here $P_{\mathrm{CR}}=E h^{2} /\left[R\left(3-3 v^{2}\right)^{1 / 2}\right]$ is the classical axial buckling load of the shell.

\subsection{General solution of the shell displacement field by a perturbation technique}

As an example, consider a simply supported cylindrical shell with radius $R=0.2 \mathrm{~m}, L=0.34163 \mathrm{~m}$ and $h=0.002$ $\mathrm{m}$. The shell material is homogeneous and isotropic with Young's modulus $E=210 \mathrm{GPa}$, Poisson's ratio $v=0.3$ and density $\rho=7850 \mathrm{~kg} / \mathrm{m}^{3}$. The vibration modes of simply-supported cylindrical shells exhibit $m$ half-waves in the axial direction and $n$ waves in the circumferential direction. For this geometry the values of the lowest natural frequencies for modes $(m, n)=(1,5)$ and $(m, N)=(1,6)$ are $3799.03 \mathrm{rad} / \mathrm{s}$ [6]. So, this cylindrical shell has two distinct vibration modes with the same natural frequency. In addition, due to the circumferential symmetry, frequencies occur in pairs. This leads to a 1:1:1:1 internal resonance. For the application of perturbation techniques, the analysis must begin by considering these four interacting modes as the modal initial solution (seed modes) in the perturbation procedure, i.e. $[5,6]$ :

$W=\left[A 1_{11}(\tau) \cos (n \theta)+B 1_{11}(\tau) \sin (n \theta)\right.$

$\left.A 2_{11}(\tau) \cos (N \theta)+B 2_{11}(\tau) \sin (N \theta)\right] \sin (m \pi \varepsilon)$

where $N=n+1$ and $A 1_{11}(\tau), \mathrm{A} 2_{11}(\tau), B 1_{11}(\tau)$ and $B 2_{11}(\tau)$ are the time dependent modal amplitudes.

By applying the perturbation procedures, as described in $[1,5,6]$, and by considering the boundary conditions for a simply supported cylindrical shell, Eq. (4), the following modal solution that accounts for the modal coupling and interaction is obtained for the transversal displacement:

$$
\begin{aligned}
& W=\sum_{i=1,3,5}^{\bar{N}_{1}} \sum_{j=1,3,5}^{\infty}\left[A 1_{i j}(\tau) \cos (\text { in } \theta)+B 1_{i j}(\tau) \sin (\text { in } \theta)\right. \\
& \left.+A 2_{i j}(\tau) \cos (i N \theta)+B 2_{i j}(\tau) \sin (i N \theta)\right] \sin (j m \pi \varepsilon) \\
& +\sum_{i=1,2,3}^{\bar{N}_{1}-1} \sum_{j=1,3,5}^{\infty}\left\{A 3_{i j}(\tau) \cos \left[\left(i N-\left(\bar{N}_{1}-i\right) n\right) \theta\right]\right. \\
& +A 4_{i j}(\tau) \cos \left[\left(i N+\left(\bar{N}_{1}-i\right) n\right) \theta\right] \\
& +B 3_{i j}(\tau) \sin \left[\left(i N-\left(\bar{N}_{1}-i\right) n\right) \theta\right] \\
& \left.+B 4_{i j}(\tau) \sin \left[\left(i N+\left(\bar{N}_{1}-i\right) n\right) \theta\right]\right\} \sin (j m \pi \varepsilon) \\
& +\sum_{\alpha=0,2,4}^{\bar{N}_{2}} \sum_{\beta=0}^{\infty}\left[A 5_{\alpha(2+6 \beta)}(\tau) \cos (\alpha n \theta)\right. \\
& +B 5_{\alpha(2+6 \beta)}(\tau) \sin (\alpha n \theta) \\
& +A 6_{\alpha(2+6 \beta)}(\tau) \cos (\alpha N \theta) \\
& \left.+B 6_{\alpha(2+6 \beta)}(\tau) \sin (\alpha N \theta)\right] \times\left\{-\frac{(3+6 \beta)}{(4+12 \beta)} \cos (6 \beta m \pi \varepsilon)\right. \\
& \left.+\cos [(2+6 \beta) m \pi \varepsilon]-\frac{(1+6 \beta)}{(4+12 \beta)} \cos [(4+6 \beta) m \pi \varepsilon]\right\} \\
& +\sum_{\alpha=1,2,3}^{\bar{N}_{2}-1} \sum_{\beta=0}^{\infty}\left\{A 7_{\alpha(2+6 \beta)}(\tau) \cos \left[\left(\alpha N-\left(\bar{N}_{2}-\alpha\right) n\right) \theta\right]\right. \\
& +B 7_{\alpha(2+6 \beta)}(\tau) \sin \left[\left(\alpha N-\left(\bar{N}_{2}-\alpha\right) n\right) \theta\right] \\
& +A 8_{\alpha(2+6 \beta)}(\tau) \cos \left[\left(\alpha N+\left(\bar{N}_{2}-\alpha\right) n\right) \theta\right] \\
& \left.\left.+B 8_{\alpha(2+6 \beta)}(\tau) \sin \left[\left(\alpha N+\left(\bar{N}_{2}-\alpha\right) n\right) \theta\right]\right]\right\} \\
& \times\left\{-\frac{(3+6 \beta)}{(4+12 \beta)} \cos (6 \beta m \pi \varepsilon)+\cos [(2+6 \beta) m \pi \varepsilon]\right. \\
& \left.-\frac{(1+6 \beta)}{(4+12 \beta)} \cos [(4+6 \beta) m \pi \varepsilon]\right\}
\end{aligned}
$$

The additional terms in Eq. (7) are due to the quadratic and cubic nonlinearities in the equations of motion. By retaining in Eq. (7) a sufficient number of terms to achieve convergence up to large vibration amplitudes [6], substituting this approximate expansion into Eq. (2) and solving analytically the partial differential equation, a consistent solution for the stress 
function $F$ is obtained. Finally, by substituting the adopted expansion for the transversal displacement together with the obtained stress function into the Eq. (1) and by applying the standard Galerkin method, a discretized system of ordinary differential equations of motion is derived.

\section{Numerical results}

The results presented here are for a cylindrical shell with physical and geometrical properties defined at section 2.2. The linear viscous damping coefficient, $\beta_{1}$, is 0.001 and the viscoelastic material damping, $\beta_{2}$, is 0.0001 . In order to understand the influence of the modal interaction on the nonlinear behavior of the shell, the three following modal expansions are obtained from Eq. (7):

1. Model $1-(\mathrm{m}, \mathrm{n})=(1,5)$

9 D.O.F $-A 1_{11}(\tau)+B 1_{11}(\tau)+A 5_{02}(\tau)+A 5_{22}(\tau)+B 5_{22}(\tau)+$ $A 1_{13}(\tau)+B 1_{13}(\tau)+A 1_{31}(\tau)+B 1_{31}(\tau)$.

2. Model $2-(\mathrm{m}, \mathrm{N})=(1,6)$

9 D.O.F $-A 2_{11}(\tau)+B 2_{11}(\tau)+A 6_{02}(\tau)+A 6_{22}(\tau)+B 6_{22}(\tau)+$ $A 2_{13}(\tau)+B 2_{13}(\tau)+A 2_{31}(\tau)+B 2_{31}(\tau)$.

3. Model 3- $(\mathrm{m}, \mathrm{n})+(\mathrm{m}, \mathrm{N})=(1,5)+(1,6)$

29 D.O.F $-A 1_{11}(\tau)+B 1_{11}(\tau)+A 2_{11}(\tau)+B 2_{11}(\tau)+A 5_{02}(\tau)+$ $A 5_{22}(\tau)+B 5_{22}(\tau)+A 6_{22}(\tau)+B 6_{22}(\tau)+\boldsymbol{A} \mathbf{7}_{12}(\tau)+B 7_{12}(\tau)+$ $A 8_{12}(\tau)+B 8_{12}(\tau)+A 1_{13}(\tau)+B 1_{13}(\tau)+A 2_{13}(\tau)+B 2_{13}(\tau)+$ $A 1_{31}(\tau)+B 1_{31}(\tau)+A 2_{31}(\tau)+B 2_{31}(\tau)+\boldsymbol{A 3}_{11}(\tau)+B \mathbf{3}_{11}(\tau)+$ $A 4_{11}(\tau)+B 4_{11}(\tau)+A 3_{21}(\tau)+B 3_{21}(\tau)+A 4_{21}(\tau)+B 4_{21}(\tau)$.

Model 1 considers the two vibrations modes with $(m$, $n)=(1,5)$ and the associated modes due to modal coupling. Model 2 is the modal expansion for the two modes with $(\mathrm{m}, \mathrm{N})=(1,6)$. Model 3 consists of the addition of models 1 and 2 plus the new modes due to their modal interaction, which are written in bold type.
In this work an axially pre-loaded cylindrical shell is analyzed. The initial pre-load of the shell is located between the critical static load, $\Gamma_{0-\mathrm{CR}}$, and the minimum post-critical load $\Gamma_{\min }$, so that a three potential well function is obtained, and, the adopted value of the preload is $\Gamma_{0}=0.50$. For this load level the shells displays five static solutions: a fundamental, two stable and two unstable post-critical solutions [6]. Both the principal and secondary instability regions - which are the most important regions in non-linear dynamic analysis - for modes $(m, n)=(1,5)$ and $(m, n)=(1,6)$, are located in the region $\Omega_{\mathrm{f}}<2$ due to the static pre-load [6]. Figure 2 displays both the parametric instability (PI) and permanent escape (PE) boundaries for different vibration modes $(m, n)$. As can be observed, other vibration modes also influence the dynamics of the shell in this forcing region. In this figure, only the modes that influence the instability boundaries are displayed [6].

In figure 2 the uncoupled modes $(m, n)=(1,5)$ and $(m, n)=(1,6)$ interact with modes $(m, n)=(1,4)$ and $(m$, $n)=(2,6)$ which influence strongly both the parametric instability and permanent escape boundaries. So, the interaction between modes $(m, n)=(1,4)$ and $(m, n)=(2$, 6) with modes $(m, n)=(1,5)$ and $(m, n)=(1,6)$ is considered. The region located below the parametric instability boundary represents combinations of harmonic load amplitude, $\Gamma_{1}$, and load excitation frequency, $\Omega_{\mathrm{f}}$, that lead to trivial solutions. The region located between the parametric instability boundary and permanent escape boundary, displays nontrivial solutions within the prebuckling safe well. This region is limited by the saddle points associated with the unstable post-critical solutions for the defined load level. The region above the permanent escape boundary, represents combinations of de $\Gamma_{1}$ e $\Omega_{\mathrm{f}}$ that generate large amplitude cross-well vibrations or small vibrations within a post-buckling well.

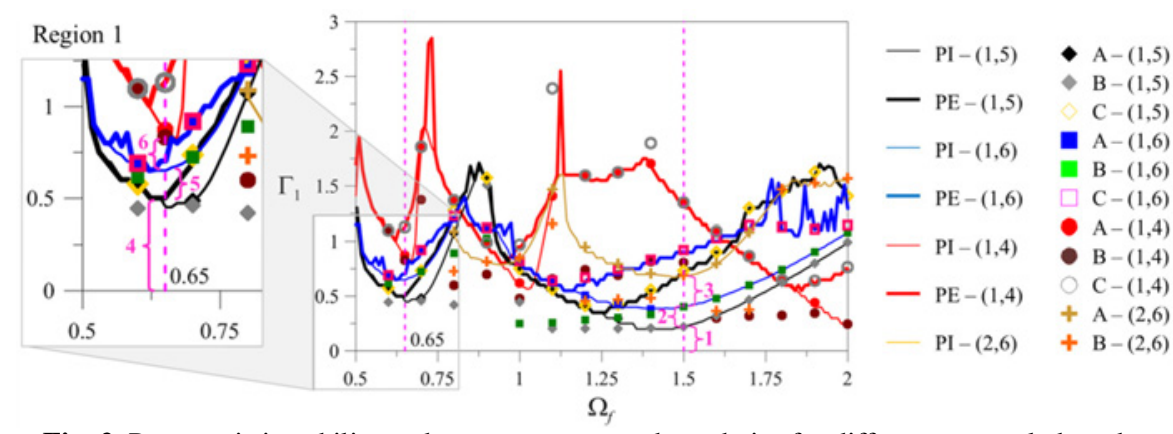

Fig. 2. Parametric instability and permanent escape boundaries for different uncoupled modes.

A typical bifurcation diagram is shown in figure 3 where the bifurcation points $\mathbf{A}, \mathbf{B}$ and $\mathbf{C}$ are identified. Point $\mathbf{A}$ represents the parametric instability point. Point B represents the point where the first saddle-node bifurcation appears. Finally, Point $\mathbf{C}$ represents escape from the pre-buckling well [6]. Points A, B and $\mathbf{C}$ for each uncoupled modes are displayed in figure 2 after an analysis of several bifurcation diagrams. So, even before the parametric instability boundary is attained, nontrivial solutions may occur due to the sub-critical perioddoubling bifurcations of each uncoupled mode.
Figure 4 display a cross section of the basin of attraction by the plane $A 1_{11}(\tau) \times A 2_{11}(\tau)$ considering $\Gamma_{0}$ $=0.50, \Omega_{\mathrm{f}}=1.50$ and $\Gamma_{1}=0.30$ (figure $4 \mathrm{a}$ ) and $\Omega_{\mathrm{f}}=0.65$ and $\Gamma_{1}=0.80$ (figure $4 \mathrm{~d}$ ). These basins of attraction were obtained considering Model 3 (coupled model). Observing the dashed vertical line at $\Omega_{0}=1.50$, it is observed that, before the saddle-node bifurcation of mode $(m, n)=(1,5)$ only the trivial solution exists, after the saddle-node bifurcations at least two solutions coexist, the $2 \mathrm{~T}$ solution of mode $(1,5)$ and the trivial solution. After the saddle-node bifurcation of mode $(1,6)$, at least 
three solutions coexists, the trivial one, the 2T solution of mode $(1,5)$ and the $2 \mathrm{~T}$ solution of mode $(1,6)$, which are uncoupled solutions. The cross sections of basins of attraction by the planes $A 1_{11}(\tau)$ x $A 2_{11}(\tau)$ allows one to observe new stable coupled solutions which are not possible to identify in the bifurcation diagrams of uncoupled modes [6]. From the phase portraits of figures $4 \mathrm{~b}$ and $4 \mathrm{e}$, it is possible to observe that there are orbits coupling the displacements of classic modes $(1,5)$ and $(1$, 6 ), which coexists with the uncoupled nontrivial solutions, two examples are shown in figures $4 \mathrm{c}$ and $4 \mathrm{f}$.

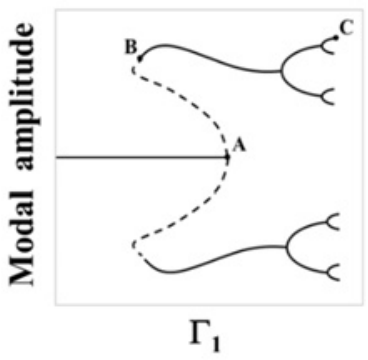

Fig. 3. Bifurcations of equilibrium solutions. ${ }^{-}$stable solutions and --- unstable solutions.

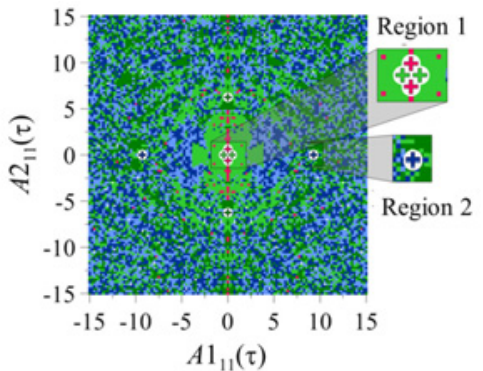

(a) $\Omega_{0}=1.50$ and $\Gamma_{1}=0.50$.

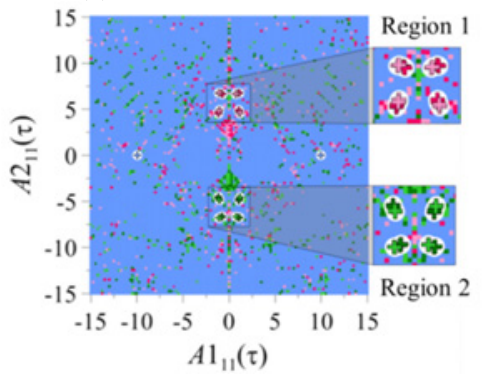

(d) $\Omega_{0}=0.65$ and $\Gamma_{1}=0.80$.

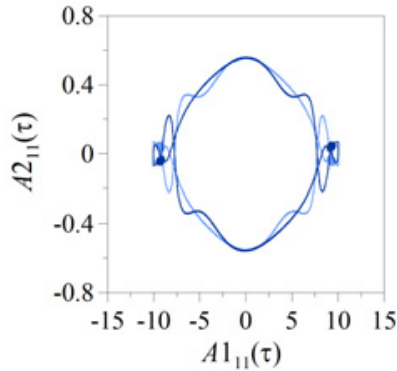

(b) coupled response

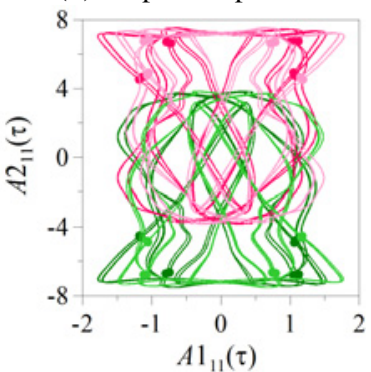

(e) coupled response

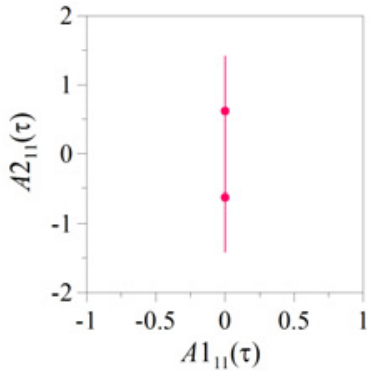

(c) uncoupled response

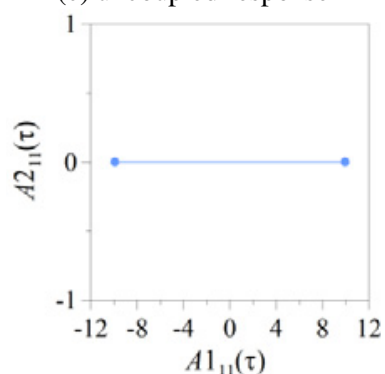

(f) uncoupled response

Fig. 4. Basin of attraction and phase portraits. Model 3. $\Gamma_{0}=0.50$.

\section{Conclusions}

In the present paper, the influence of the nonlinear interaction among four neighbor modes with the same natural frequency, leading to a 1:1:1:1 internal resonance, on the stability of a cylindrical shell under axial load is studied. To model the shell, the Donnell nonlinear shallow shell theory is used. Based on a perturbation procedure, a general solution for the transversal displacement field considering the interaction among these modes is obtained which satisfies the boundary conditions of a simply-supported shell. Precise low dimensional models considering or not the modal interaction are derived and results are compared. The results show that the modal interaction among these modes with the same or nearly the same natural frequencies, a phenomenon observed in many practical shell geometries, may lead to many unsafe nonlinear phenomena, such as unstable bifurcations and jumps. Consequently, this must be considered for a safe shell design in a dynamic environment. Of course, due to the complexity of the nonlinear shell vibration observed in the present work and the dense frequency spectrum of shell structures, additional research must be conducted to unveil the influence of modal interaction in the nonlinear shell dynamics. This may influence significantly the dynamic integrity of the shell in a dynamic environment [7].

\section{References}

1. P. B. Gonçalves, R. C. Batista, J. Sound Vib. 127 (1988) 133-143

2. A. A. Popov, J. M. T. Thompson, F. A. McRobie, J. Sound Vib. 209 (1998) 163-186

3. P. B. Gonçalves, Z. J. G N. Del Prado, Comp. Struct. 82 (2004) 2621-2634

4. M. Amabili, Nonlinear Dyn. 69 (2012) 755-770

5. P. B. Gonçalves, F. M. A Silva, Z. J. G. N. Del Prado, J. Sound Vib. 315 (2008) 641-663

6. L. Rodrigues, F. M. A. Silva, P. B. Gonçalves, Z. J. G N. Del Prado, Thin Wall. Struct. on-line first (2013) 1-15

7. P. B. Gonçalves, F. M. A. Silva, G. Rega and S. Lenci, Nonlinear Dynamics, 63 (2011) 61-82 